NOTICE: this is the author's version of a work that was accepted for publication in Applied Mathematics and Computations. Changes resulting from the publishing process, such as peer review, editing, corrections, structural formatting, and other quality control mechanisms may not be reflected in this document. Changes may have been made to this work since it was submitted for publication. A definitive version was subsequently published in Applied Mathematics and Computations, 217, 14, 2011.

DOI: 10.1016/j.amc.2011.01.039 


\title{
Robust Suboptimal Control of Nonlinear Systems
}

\author{
R. Loxton ${ }^{\mathrm{a}, *}, \mathrm{~K}$. L. Teo ${ }^{\mathrm{a}}$, V. Rehbock ${ }^{\mathrm{a}}$ \\ ${ }^{a}$ Department of Mathematics and Statistics, Curtin University, Perth, Australia
}

\begin{abstract}
In this paper, we consider a nonlinear dynamic system with uncertain parameters. Our goal is to choose a control function for this system that balances two competing objectives: (i) the system should operate efficiently; and (ii) the system's performance should be robust with respect to changes in the uncertain parameters. With this in mind, we introduce an optimal control problem with a cost function penalizing both the system cost (a function of the final state reached by the system) and the system sensitivity (the derivative of the system cost with respect to the uncertain parameters). We then show that the system sensitivity can be computed by solving an auxiliary initial value problem. This result allows one to convert the optimal control problem into a standard Mayer problem, which can be solved directly using conventional techniques. We illustrate this approach by solving two example problems using the software MISER3.
\end{abstract}

Keywords: Robust control, Optimal control, Nonlinear systems, System sensitivity

\section{Introduction}

There are many excellent methods for solving optimal control problems numerically - for example, the control parameterization method $[8,12,13]$, the state discretization method $[2,5,7,14]$, and the sequential gradient restoration method $[4,9]$. These methods can be used to find an open-loop control function - the so-called optimal control - that minimizes a given cost function subject to a dynamic system.

The dynamic system in an optimal control problem is usually only an approximation of the real system under consideration. Thus, it is imperative that the optimal control be robust with respect to modelling errors. In other words, the performance of the real system when the optimal control is applied should be similar to the theoretical performance predicted by the model. Unfortunately,

\footnotetext{
* Corresponding author

Email addresses: r.loxton@curtin.edu.au (R. Loxton), k.l.teo@curtin.edu.au (K. L. Teo), v.rehbock@curtin.edu.au (V. Rehbock)
} 
this is not always the case - the optimal control gives the best theoretical performance, but it may also make the system highly susceptible to random noise, disturbances, and uncertainties.

Therefore, in practice there is always a tradeoff between performance and robustness. The most efficient control policy is probably not robust, while an overly robust control policy may be too conservative. Previously, Rehbock, Teo, and Jennings modelled this tradeoff as an optimal control problem in which the control needs to be chosen to minimize the sum of system cost and system sensitivity [11]. Solving this problem yields a control scheme that is both efficient and robust. However, this optimal control problem is rather complex and can only be solved using specialized techniques.

The technique developed in [11] is based on control parameterization, which involves approximating the control by a piecewise constant function. Applying this approximation technique yields an approximate nonlinear programming problem. This approximate problem has a cost function whose gradient is computed by first integrating two dynamic systems forward in time (one of these is the original dynamic system), and then integrating another two dynamic systems backwards in time (the so-called costate systems). These costate systems are extremely complex. Furthermore, they are coupled with the original dynamic system, so integrating them backwards is a very difficult task. Consequently, the nonlinear programming problem that approximates the optimal control problem in [11] is very difficult to solve.

In this paper, we consider an optimal control problem that is similar to the one formulated in [11]. We prove that the system sensitivity can be computed by solving an auxiliary initial value problem. We then use this result to convert the original optimal control problem into a standard Mayer optimal control problem, which can be solved readily using existing analytical or numerical techniques. By approaching the problem in this way, we can avoid the complex procedure developed in [11]. This is a major advantage that makes our new approach more flexible and powerful than the one proposed in [11].

\section{Problem Formulation}

We consider control systems of the following form:

$$
\dot{\boldsymbol{x}}(t)=\boldsymbol{f}(t, \boldsymbol{x}(t), \boldsymbol{u}(t), \boldsymbol{\sigma}), \quad t \in[0, T],
$$

and

$$
\boldsymbol{x}(0)=\boldsymbol{\varphi}(\boldsymbol{\sigma}),
$$

where $T>0$ is a given terminal time; $\boldsymbol{x}(t) \in \mathbb{R}^{n}$ is the system's state at time $t ; \boldsymbol{u}(t) \in \mathbb{R}^{r}$ is the control input at time $t ; \boldsymbol{\sigma} \in \mathbb{R}^{m}$ is a vector of uncertain parameters; and $\boldsymbol{f}: \mathbb{R} \times \mathbb{R}^{n} \times \mathbb{R}^{r} \times \mathbb{R}^{m} \rightarrow \mathbb{R}^{n}$ and $\boldsymbol{\varphi}: \mathbb{R}^{m} \rightarrow \mathbb{R}^{n}$ are given functions.

Let

$$
\mathcal{W} \triangleq\left\{\boldsymbol{w} \in \mathbb{R}^{r}: a_{i} \leq w_{i} \leq b_{i}, i=1, \ldots, r\right\}
$$


where $a_{i}$ and $b_{i}, i=1, \ldots, r$, are given real numbers such that $a_{i}<b_{i}$. Any measurable function $\boldsymbol{u}:[0, T] \rightarrow \mathbb{R}^{r}$ such that $\boldsymbol{u}(t) \in \mathcal{W}$ for all $t \in[0, T]$ is called an admissible control. Let $\mathcal{U}$ denote the class of all such admissible controls.

We assume that the control system (1)-(2) satisfies the following two conditions.

Assumption 1. Both $\boldsymbol{f}$ and $\boldsymbol{\varphi}$ are continuously differentiable, and $\boldsymbol{f}$ is twice continuously differentiable.

Assumption 2. There exists a real number $L_{1}>0$ such that

$$
|\boldsymbol{f}(\eta, \boldsymbol{v}, \boldsymbol{w}, \boldsymbol{z})|_{n} \leq L_{1}\left(1+|\boldsymbol{v}|_{n}+|\boldsymbol{z}|_{m}\right), \quad(\eta, \boldsymbol{v}, \boldsymbol{w}, \boldsymbol{z}) \in[0, T] \times \mathbb{R}^{n} \times \mathcal{W} \times \mathbb{R}^{m},
$$

where $|\cdot|_{n}$ and $|\cdot|_{m}$ denote the Euclidean norms in $\mathbb{R}^{n}$ and $\mathbb{R}^{m}$, respectively.

Let $\boldsymbol{x}(\cdot \mid \boldsymbol{u}, \boldsymbol{\sigma}):[0, T] \rightarrow \mathbb{R}^{n}$ denote the solution of (1)-(2) corresponding to the control $\boldsymbol{u} \in \mathcal{U}$ and the parameter vector $\boldsymbol{\sigma} \in \mathbb{R}^{m}$. Assumptions 1 and 2 ensure that $\boldsymbol{x}(\cdot \mid \boldsymbol{u}, \boldsymbol{\sigma})$ exists and is unique (see Theorem 3.3.3 of [1]). Note that $\boldsymbol{x}(\cdot \mid \boldsymbol{u}, \boldsymbol{\sigma})$ is an absolutely continuous function satisfying the dynamics (1) almost everywhere and the initial condition (2).

If the model parameters are fixed, then the behaviour of system (1)-(2) is completely determined by the control. Our goal is to choose the control appropriately so that the system performs as efficiently as possible. To this end, we assign a system cost - which depends on the final state reached by the system - to each admissible control. More specifically, given the parameter vector $\boldsymbol{\sigma} \in \mathbb{R}^{m}$, we define a cost function $G(\cdot \mid \boldsymbol{\sigma}): \mathcal{U} \rightarrow \mathbb{R}$ as follows:

$$
G(\boldsymbol{u} \mid \boldsymbol{\sigma}) \triangleq \Phi(\boldsymbol{x}(T \mid \boldsymbol{u}, \boldsymbol{\sigma})), \quad \boldsymbol{u} \in \mathcal{U},
$$

where $\Phi: \mathbb{R}^{n} \rightarrow \mathbb{R}$ is a given function. Obviously, the lower the cost, the better the control.

Note that the cost function in [11] includes an integral term measuring the system running cost. For simplicity, we have omitted this term from (3). However, this isn't a serious restriction, as an integral term can be easily incorporated into (3) by augmenting the dynamics with an additional state variable (see Chapter 8 of [1]).

We assume that the following condition is satisfied.

Assumption 3. The function $\Phi$ is twice continuously differentiable.

In practice, the model parameters are usually not known exactly and thus need to be estimated. We suppose that $\hat{\boldsymbol{\sigma}} \in \mathbb{R}^{m}$ is a given vector whose components are nominal estimates of the model parameters in (1)-(2). By using conventional optimal control methods, we can determine a control $\boldsymbol{u}^{*}$ that minimizes the cost function $G(\cdot \mid \hat{\boldsymbol{\sigma}})$. An important issue that then arises is whether system performance under the control $\boldsymbol{u}^{*}$ is sensitive to errors in $\hat{\boldsymbol{\sigma}}$. In particular, how does the system perform if the true parameters differ slightly from the nominal estimates? Is the system still efficient when the control $\boldsymbol{u}^{*}$ is applied? 
We define the system sensitivity corresponding to a control $\boldsymbol{u} \in \mathcal{U}$ as

$$
\frac{\partial G(\boldsymbol{u} \mid \hat{\boldsymbol{\sigma}})}{\partial \boldsymbol{\sigma}}\left[\frac{\partial G(\boldsymbol{u} \mid \hat{\boldsymbol{\sigma}})}{\partial \boldsymbol{\sigma}}\right]^{\top} .
$$

(We will show in the next section that these partial derivatives exist.) The system sensitivity measures the rate at which the system cost changes in response to small changes in the model parameters. Hence, a low system sensitivity indicates that the system is robust.

The ideal control function is one that simultaneously minimizes both system cost and system sensitivity. Such a control, however, is unlikely to exist. Thus, we need a compromise, and this leads to the following optimal control problem.

Problem P. Given the nominal parameter vector $\hat{\boldsymbol{\sigma}} \in \mathbb{R}^{m}$ and a real number $\alpha \geq 0$, choose an admissible control to minimize

$$
J_{\alpha}(\boldsymbol{u} \mid \hat{\boldsymbol{\sigma}}) \triangleq G(\boldsymbol{u} \mid \hat{\boldsymbol{\sigma}})+\alpha \frac{\partial G(\boldsymbol{u} \mid \hat{\boldsymbol{\sigma}})}{\partial \boldsymbol{\sigma}}\left[\frac{\partial G(\boldsymbol{u} \mid \hat{\boldsymbol{\sigma}})}{\partial \boldsymbol{\sigma}}\right]^{\top}, \quad \boldsymbol{u} \in \mathcal{U} .
$$

The relative importance of each term in $J_{\alpha}(\cdot \mid \hat{\boldsymbol{\sigma}})$ can be adjusted through the weight $\alpha$. When $\alpha=0$, the sensitivity term disappears and Problem P only considers system performance, disregarding robustness. In this case, Problem P is a standard Mayer optimal control problem and can be solved using one of the numerical methods mentioned in the introduction. Alternatively, it may be possible to solve Problem P using an analytical technique such as the Pontryagin minimum principle.

We are most interested in Problem $\mathrm{P}$ when $\alpha>0$. In this case, conventional optimal control techniques are not directly applicable because the cost function $J_{\alpha}(\cdot \mid \hat{\boldsymbol{\sigma}})$ contains a non-standard sensitivity term. The aim of this paper is to present a method for transforming Problem $\mathrm{P}$ into a standard problem. To this end, we will prove in the next section that the system sensitivity is defined for each admissible control (this ensures that Problem P makes sense). Incidentally, this result also reveals how the system sensitivity can be computed efficiently.

\section{Computing the System Sensitivity}

Consider the following auxiliary dynamic system:

$$
\begin{aligned}
& \dot{\boldsymbol{\psi}}^{k}(t)=\frac{\partial \boldsymbol{f}(t, \boldsymbol{x}(t \mid \boldsymbol{u}, \hat{\boldsymbol{\sigma}}), \boldsymbol{u}(t), \hat{\boldsymbol{\sigma}})}{\partial \boldsymbol{x}} \boldsymbol{\psi}^{k}(t)+\frac{\partial \boldsymbol{f}(t, \boldsymbol{x}(t \mid \boldsymbol{u}, \hat{\boldsymbol{\sigma}}), \boldsymbol{u}(t), \hat{\boldsymbol{\sigma}})}{\partial \sigma_{k}}, \\
& t \in[0, T], \quad k=1, \ldots, m,
\end{aligned}
$$

and

$$
\boldsymbol{\psi}^{k}(0)=\frac{\partial \boldsymbol{\varphi}(\hat{\boldsymbol{\sigma}})}{\partial \sigma_{k}}, \quad k=1, \ldots, m
$$


where $\boldsymbol{u} \in \mathcal{U}$ is a given admissible control. Let $\boldsymbol{\psi}^{k}(\cdot \mid \boldsymbol{u}), k=1, \ldots, m$, denote the solution of (4)-(5) corresponding to $\boldsymbol{u} \in \mathcal{U}$. Assumptions 1 and 2 ensure that such a solution exists and is unique.

We have the following important result, the proof of which is given in the appendix.

Theorem 1. For each admissible control $\boldsymbol{u} \in \mathcal{U}$,

$$
\frac{\partial \boldsymbol{x}(t \mid \boldsymbol{u}, \hat{\boldsymbol{\sigma}})}{\partial \sigma_{k}}=\boldsymbol{\psi}^{k}(t \mid \boldsymbol{u}), \quad t \in[0, T], \quad k=1, \ldots, m
$$

According to Theorem 1, the state is differentiable with respect to the uncertain parameters, and the partial derivatives of the state with respect to these uncertain parameters satisfy the auxiliary system (4)-(5). We now use this result to derive a formula for the system sensitivity in Problem P.

Theorem 2. For each admissible control $\boldsymbol{u} \in \mathcal{U}$,

$$
\frac{\partial G(\boldsymbol{u} \mid \hat{\boldsymbol{\sigma}})}{\partial \boldsymbol{\sigma}}\left[\frac{\partial G(\boldsymbol{u} \mid \hat{\boldsymbol{\sigma}})}{\partial \boldsymbol{\sigma}}\right]^{\top}=\sum_{k=1}^{m}\left[\frac{\partial \Phi(\boldsymbol{x}(T \mid \boldsymbol{u}, \hat{\boldsymbol{\sigma}}))}{\partial \boldsymbol{x}} \psi^{k}(T \mid \boldsymbol{u})\right]^{2}
$$

Proof. Let $\boldsymbol{u} \in \mathcal{U}$ be an admissible control. By Theorem 1,

$$
\frac{\partial \boldsymbol{x}(T \mid \boldsymbol{u}, \hat{\boldsymbol{\sigma}})}{\partial \sigma_{k}}=\boldsymbol{\psi}^{k}(T \mid \boldsymbol{u}), \quad k=1, \ldots, m
$$

Thus, differentiating $G(\boldsymbol{u} \mid \hat{\boldsymbol{\sigma}})=\Phi(\boldsymbol{x}(T \mid \boldsymbol{u}, \hat{\boldsymbol{\sigma}}))$ with respect to $\sigma_{k}$ yields

$$
\frac{\partial G(\boldsymbol{u} \mid \hat{\boldsymbol{\sigma}})}{\partial \sigma_{k}}=\frac{\partial \Phi(\boldsymbol{x}(T \mid \boldsymbol{u}, \hat{\boldsymbol{\sigma}}))}{\partial \boldsymbol{x}} \boldsymbol{\psi}^{k}(T \mid \boldsymbol{u}), \quad k=1, \ldots, m
$$

Hence,

$$
\frac{\partial G(\boldsymbol{u} \mid \hat{\boldsymbol{\sigma}})}{\partial \boldsymbol{\sigma}}\left[\frac{\partial G(\boldsymbol{u} \mid \hat{\boldsymbol{\sigma}})}{\partial \boldsymbol{\sigma}}\right]^{\top}=\sum_{k=1}^{m}\left[\frac{\partial G(\boldsymbol{u} \mid \hat{\boldsymbol{\sigma}})}{\partial \sigma_{k}}\right]^{2}=\sum_{k=1}^{m}\left[\frac{\partial \Phi(\boldsymbol{x}(T \mid \boldsymbol{u}, \hat{\boldsymbol{\sigma}}))}{\partial \boldsymbol{x}} \boldsymbol{\psi}^{k}(T \mid \boldsymbol{u})\right]^{2},
$$

as required.

Theorem 2 shows that the system sensitivity can be computed by solving the initial value problem (4)-(5). We will use this result in the next section to convert Problem P into a Mayer optimal control problem.

\section{Transforming Problem P into Mayer form}

By virtue of Theorem 2, we have

$$
J_{\alpha}(\boldsymbol{u} \mid \hat{\boldsymbol{\sigma}})=\Phi(\boldsymbol{x}(T \mid \boldsymbol{u}, \hat{\boldsymbol{\sigma}}))+\alpha \sum_{k=1}^{m}\left[\frac{\partial \Phi(\boldsymbol{x}(T \mid \boldsymbol{u}, \hat{\boldsymbol{\sigma}}))}{\partial \boldsymbol{x}} \boldsymbol{\psi}^{k}(T \mid \boldsymbol{u})\right]^{2}, \quad \boldsymbol{u} \in \mathcal{U}
$$


This equation expresses $J_{\alpha}(\cdot \mid \hat{\boldsymbol{\sigma}})$ in terms of the solutions of the state and auxiliary systems at the terminal time. Since these systems are coupled (the state appears in the right-hand side of the auxiliary system), we can combine them to form an expanded dynamic system. This expanded system can be viewed as a new state system in which $\boldsymbol{x}$ and $\boldsymbol{\psi}^{k}, k=1, \ldots, m$, are the new state variables. Equation (6) shows that $J_{\alpha}(\cdot \mid \hat{\boldsymbol{\sigma}})$ is a Mayer function for this new system.

It should now be clear that Problem $\mathrm{P}$ is equivalent to the following optimal control problem.

Problem $\tilde{\mathbf{P}}$. Given the nominal parameter vector $\hat{\boldsymbol{\sigma}} \in \mathbb{R}^{m}$ and a real number $\alpha \geq 0$, choose an admissible control to minimize

$$
J_{\alpha}(\boldsymbol{u} \mid \hat{\boldsymbol{\sigma}})=\Phi(\boldsymbol{x}(T \mid \boldsymbol{u}, \hat{\boldsymbol{\sigma}}))+\alpha \sum_{k=1}^{m}\left[\frac{\partial \Phi(\boldsymbol{x}(T \mid \boldsymbol{u}, \hat{\boldsymbol{\sigma}}))}{\partial \boldsymbol{x}} \boldsymbol{\psi}^{k}(T \mid \boldsymbol{u})\right]^{2}, \quad \boldsymbol{u} \in \mathcal{U}
$$

The dynamic system for Problem $\tilde{\mathrm{P}}$ consists of (1)-(2) and (4)-(5), not just (1)(2). Hence, Problem $\tilde{\mathrm{P}}$ is a standard Mayer optimal control problem and can be solved using conventional techniques. In particular, since $\boldsymbol{f}$ and $\Phi$ are twice continuously differentiable (recall Assumptions 1 and 3), Problem $\tilde{\mathrm{P}}$ can be solved using techniques based on nonlinear programming (for example, control parameterization methods $[8,12,13])$.

We emphasize here that (in principle) any conventional optimal control technique can be applied to Problem $\tilde{\mathrm{P}}$. Consequently, Problem $\tilde{\mathrm{P}}$ is much easier to solve than Problem P. Indeed, the only method capable of solving Problem P is the one described in [11], and this method involves solving a very complicated nonlinear programming problem. Computing the cost function's gradient for this nonlinear programming problem involves integrating four dynamic systems (two of them backwards in time). We can avoid this by first transforming Problem P into Problem $\tilde{P}$.

\section{Examples}

For illustration, we consider two examples. Problem $\tilde{\mathrm{P}}$ for each example was solved using MISER3 [6], a Fortran program capable of solving a wide variety of dynamic optimization problems, including those with state constraints. It's important to note that MISER3 can solve Problem $\tilde{P}$ directly, but not Problem P. To solve Problem P using MISER3, one needs to significantly modify MISER3's internal engine [11].

\subsection{Modified Rayleigh System}

For our first example, we modify the Rayleigh system in [10] to obtain the dynamics

$$
\begin{array}{ll}
\dot{x}_{1}(t)=x_{2}(t), & t \in[0,10], \\
\dot{x}_{2}(t)=-x_{1}(t)+\left[1.4+u(t)-0.14 x_{2}^{2}(t)\right] x_{2}(t)+4 u(t), & t \in[0,10],
\end{array}
$$


and

$$
\begin{aligned}
& x_{1}(0)=-5+\sigma, \\
& x_{2}(0)=-5,
\end{aligned}
$$

where $u$ is a control function and $\sigma$ is a model parameter. The cost function for this system is

$$
G=\int_{0}^{10}\left(x_{1}^{2}(t)+u^{2}(t)\right) d t .
$$

Because $G$ is expressed as an integral, we cannot calculate the system sensitivity using Theorem 2. Thus, we introduce a new state variable $x_{3}$ with dynamics

$$
\dot{x}_{3}(t)=x_{1}^{2}(t)+u^{2}(t), \quad t \in[0,10],
$$

and

$$
x_{3}(0)=0 .
$$

Consequently, the cost function becomes

$$
G=x_{3}(10) .
$$

Suppose that the nominal value of $\sigma$ is zero (that is, $\hat{\sigma}=0$ ). Our optimal control problem is to choose $u$ to minimize

$$
J_{\alpha}=G+\alpha\left[\frac{\partial G}{\partial \sigma}\right]^{2},
$$

where $\alpha \geq 0$, subject to the dynamic system consisting of equations (7)-(10).

We can convert this problem into Mayer form by applying the procedure discussed in Section 4. First, define the auxiliary system for this problem:

$$
\begin{aligned}
& \dot{\psi}_{1}(t)=\psi_{2}(t), \quad t \in[0,10], \\
& \dot{\psi}_{2}(t)=-\psi_{1}(t)+\left[1.4+u(t)-0.42 x_{2}^{2}(t)\right] \psi_{2}(t), \quad t \in[0,10], \\
& \dot{\psi}_{3}(t)=2 x_{1}(t) \psi_{1}(t), \quad t \in[0,10],
\end{aligned}
$$

and

$$
\begin{aligned}
& \psi_{1}(0)=1, \\
& \psi_{2}(0)=0, \\
& \psi_{3}(0)=0 .
\end{aligned}
$$

It then follows from Theorem 2 that

$$
J_{\alpha}=x_{3}(10)+\alpha \psi_{3}^{2}(10) .
$$

Thus, our optimal control problem becomes: choose $u$ to minimize (13) subject to the dynamic system consisting of equations (7)-(12). This problem is a 

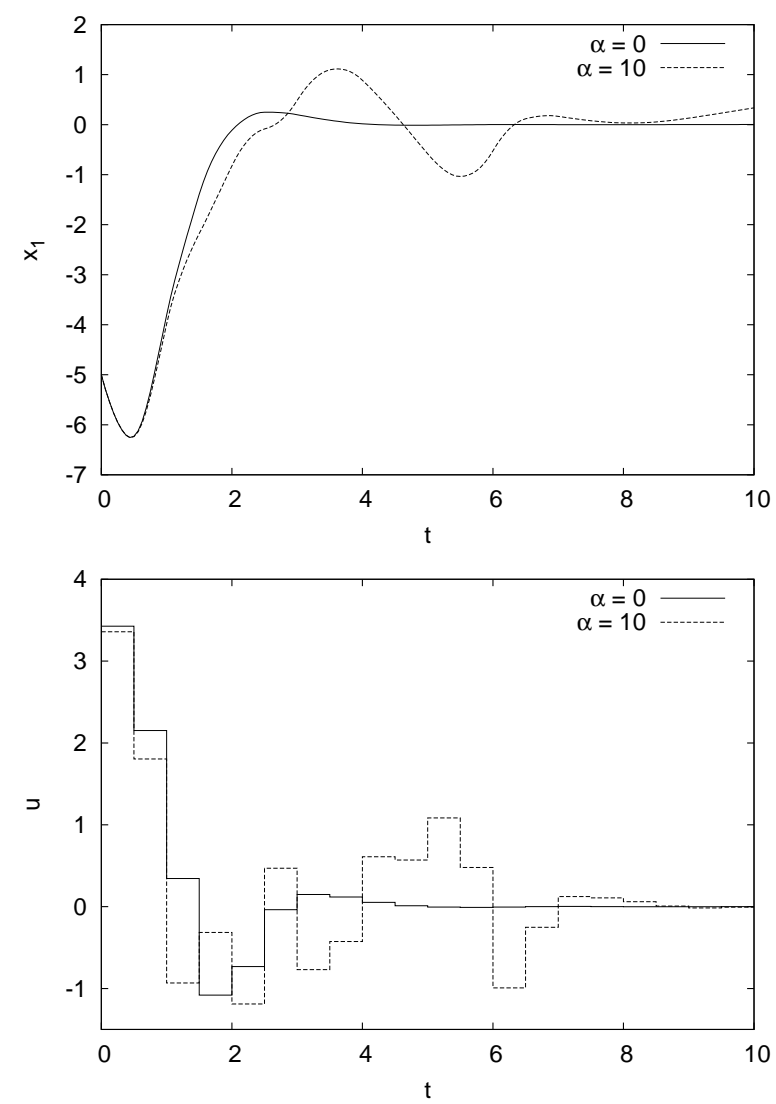

Figure 1: Optimal system trajectories and optimal controls in Example 5.1.

standard Mayer optimal control problem. We solve it with MISER3 for $\alpha=0$ and $\alpha=10$. When $\alpha=0$, system sensitivity is not penalized in (13). The optimal system cost in this case is 44.0099 and the system sensitivity is 99.6791 . When $\alpha=10$, equation (13) penalizes both system cost and system sensitivity. The optimal cost in this case is 50.0009 and the system sensitivity is zero (to four decimal places). As expected, some performance is sacrificed when $\alpha=10$, but the advantage is that system sensitivity is much lower. The optimal controls and optimal state trajectories are displayed in Figure 1.

We now test the robustness of the original system under the controls in Figure 1. To do this, we perturb the nominal value of $\sigma$ by a small amount and then simulate the system under each control. The costs of each control for different values of the model parameter are shown in Figure 2. It's clear from this figure that the control obtained by solving the problem with $\alpha=10$ is very robust. The cost of the system under this control hardly changes when $\sigma$ is changed. 


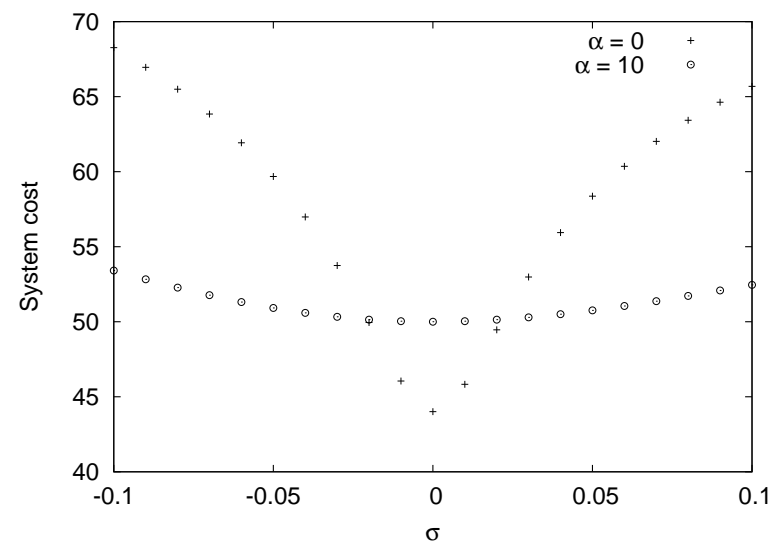

Figure 2: The cost of each optimal control for different values of the model parameter in Example 5.1.

\subsection{A Robotic Manipulator}

According to [3], a two-link planar robotic manipulator can be described by the following dynamics:

$$
\begin{aligned}
\dot{x}_{1}= & x_{2}, \\
\dot{x}_{2}= & \frac{1}{\sin ^{2} x_{3}+m_{1} / m_{2}}\left\{\sin x_{3}\left[\frac{l_{2}}{l_{1}}\left(x_{2}+x_{4}\right)^{2}+x_{2}^{2} \cos x_{3}\right]\right. \\
& \left.\quad+\frac{1}{m_{2} l_{1} l_{2}}\left[\frac{l_{2}}{l_{1}}\left(u_{1}-u_{2}\right)-u_{2} \cos x_{3}\right]\right\} \\
\dot{x}_{3}= & x_{4}, \\
\dot{x}_{4}= & \frac{1}{\sin ^{2} x_{3}+m_{1} / m_{2}}\left\{-\sin x_{3}\left[\left(\cos x_{3}+l_{2} / l_{1}\right)\left(x_{2}+x_{4}\right)^{2}+x_{2}^{2} \cos x_{3}\right.\right. \\
& \left.+\frac{l_{1}}{l_{2}}\left(1+m_{1} / m_{2}\right) x_{2}^{2}\right]+\frac{1}{m_{2} l_{1} l_{2}}\left[-u_{1}\left(\cos x_{3}+l_{2} / l_{1}\right)+2 u_{2} \cos x_{2}\right. \\
& \left.\left.+\frac{l_{2}}{l_{1}} u_{2}+\frac{l_{1}}{l_{2}}\left(1+m_{1} / m_{2}\right) u_{2}\right]\right\},
\end{aligned}
$$

and

$$
\begin{aligned}
& x_{1}(0)=0.75 \pi, \\
& x_{2}(0)=0, \\
& x_{3}(0)=0.25 \pi, \\
& x_{4}(0)=0,
\end{aligned}
$$

where $l_{1}$ and $l_{2}$ are the lengths of the links, $m_{1}$ and $m_{2}$ are the masses attached to the links, and $u_{1}$ and $u_{2}$ are control functions. We suppose that the following 

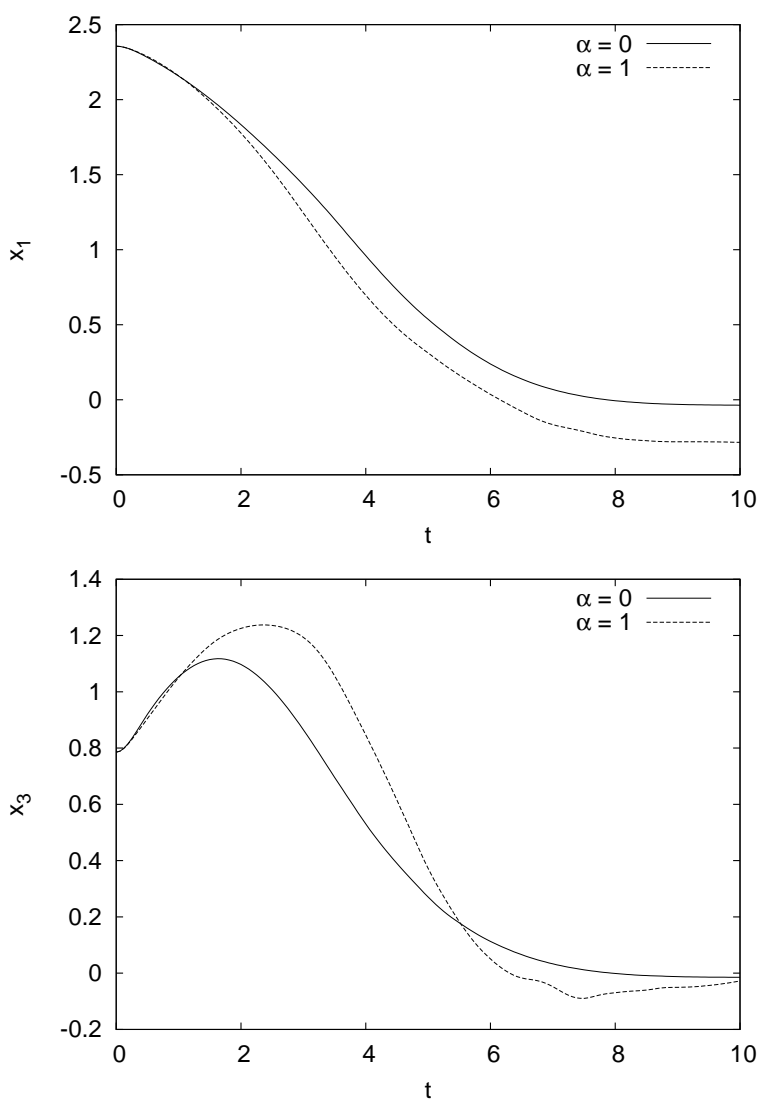

Figure 3: The optimal system trajectories in Example 5.2.

nominal values are given for the parameters $m_{1}, m_{2}, l_{1}$, and $l_{2}$ :

$$
\hat{m}_{1}=\hat{m}_{2}=\hat{l}_{1}=\hat{l}_{2}=1 \text {. }
$$

Furthermore, we suppose that the time horizon here is $[0,10]$. Our aim is to drive this system to the origin using minimal control effort. To this end, we define the system cost as

$$
\begin{aligned}
G=x_{1}^{2}(10) & +x_{2}^{2}(10)+x_{3}^{2}(10)+x_{4}^{2}(10) \\
& +\int_{0}^{10}\left\{x_{1}^{2}(t)+x_{2}^{2}(t)+x_{3}^{2}(t)+x_{4}^{2}(t)+0.1 u_{1}^{2}(t)+0.1 u_{2}^{2}(t)\right\} d t .
\end{aligned}
$$

As in Example 5.1, we introduce an extra state variable $x_{5}$ with dynamics

$$
\dot{x}_{5}=x_{1}^{2}+x_{2}^{2}+x_{3}^{2}+x_{4}^{2}+0.1 u_{1}^{2}+0.1 u_{2}^{2}
$$

and

$$
x_{5}(0)=0 .
$$



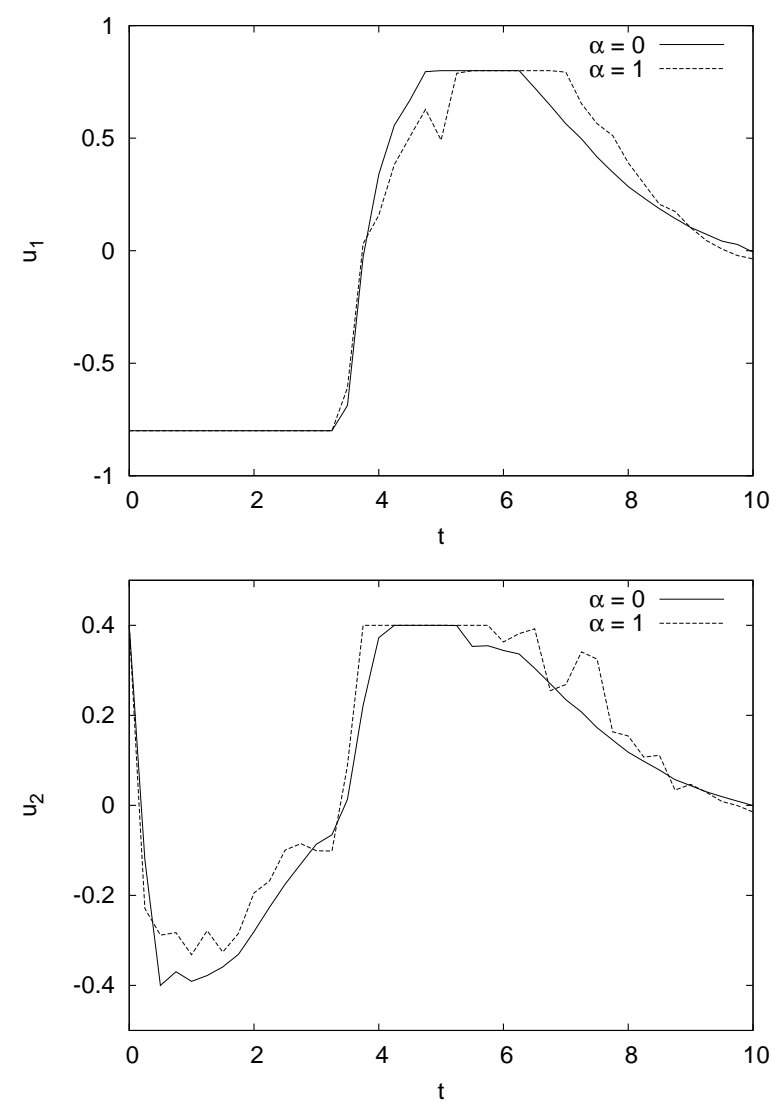

Figure 4: The optimal controls in Example 5.2.

Then the system cost can be rewritten as follows:

$$
G=x_{1}^{2}(10)+x_{2}^{2}(10)+x_{3}^{2}(10)+x_{4}^{2}(10)+x_{5}(10) .
$$

Therefore, our optimal control problem is to choose $u_{1}$ and $u_{2}$ to minimize

$$
G+\alpha\left[\frac{\partial G}{\partial m_{1}}\right]^{2}+\alpha\left[\frac{\partial G}{\partial m_{2}}\right]^{2}+\alpha\left[\frac{\partial G}{\partial l_{1}}\right]^{2}+\alpha\left[\frac{\partial G}{\partial l_{2}}\right]^{2},
$$

where $\alpha \geq 0$ is a weight, subject to the above dynamic system. We transform this problem according to the procedure in Section 4 (we omit the details for simplicity). We then solve it with MISER3 for $\alpha=0$ and $\alpha=1$. For $\alpha=0$, the optimal cost is 19.5323 and the system sensitivity is $2.0567 \times 10^{2}$. For $\alpha=1$, the optimal cost is 20.3590 and the system sensitivity is $5.8228 \times 10^{-4}$. The optimal state trajectories and optimal controls are displayed in Figures 3 and 4.

We test the robustness of the controls by perturbing the parameters and then simulating the system. Some results are shown in Table 1. This table 


\begin{tabular}{clc}
\hline & \multicolumn{2}{c}{ System Cost } \\
\cline { 2 - 3 } Model Parameters & $\alpha=0$ & $\alpha=1$ \\
\hline$m_{1}=0.97, m_{2}=1.03, l_{1}=0.97, l_{2}=1.03$ & 23.5947 & 22.9365 \\
$m_{1}=1.03, m_{2}=0.97, l_{1}=1.03, l_{2}=0.97$ & 24.9172 & 23.5462 \\
$m_{1}=1.05, m_{2}=0.95, l_{1}=1.05, l_{2}=0.95$ & 32.8406 & 28.6188 \\
$m_{1}=0.95, m_{2}=1.05, l_{1}=0.95, l_{2}=1.05$ & 28.5583 & 26.3356 \\
$m_{1}=0.93, m_{2}=1.07, l_{1}=0.93, l_{2}=0.97$ & 34.3028 & 30.4006 \\
\hline
\end{tabular}

Table 1: The system cost for different values of the model parameters in Example 5.2.

shows that the performance of the system is highly sensitive to the model parameters. Nevertheless, the control obtained by solving the problem with $\alpha=1$ is clearly more robust because its system cost is superior when the parameters are perturbed.

\section{Appendix A. Proof of Theorem 1}

Let $\boldsymbol{u} \in \mathcal{U}$ and $k \in\{1, \ldots, m\}$ be arbitrary but fixed. To prove the theorem, we need to show that

$$
\lim _{\delta \rightarrow 0} \frac{\boldsymbol{x}\left(t \mid \boldsymbol{u}, \hat{\boldsymbol{\sigma}}+\delta \boldsymbol{e}^{k}\right)-\boldsymbol{x}(t \mid \boldsymbol{u}, \hat{\boldsymbol{\sigma}})}{\delta}=\boldsymbol{\psi}^{k}(t \mid \boldsymbol{u}), \quad t \in[0, T]
$$

where $\boldsymbol{e}^{k}$ is the $k$ th unit vector in $\mathbb{R}^{m}$. We will prove this equation in five steps.

\section{Step 1. Preliminaries}

For each real number $\delta \in \mathbb{R}$, let $\boldsymbol{x}^{\delta}$ denote the function $\boldsymbol{x}\left(\cdot \mid \boldsymbol{u}, \hat{\boldsymbol{\sigma}}+\delta \boldsymbol{e}^{k}\right)$ (this notation will not cause confusion because $k, \boldsymbol{u}$, and $\hat{\boldsymbol{\sigma}}$ are fixed). Furthermore, for each $\delta \in \mathbb{R}$, define a corresponding function $\boldsymbol{v}^{\delta}:[0, T] \rightarrow \mathbb{R}^{n}$ as follows:

$$
\boldsymbol{v}^{\delta}(t) \triangleq \boldsymbol{x}^{\delta}(t)-\boldsymbol{x}^{0}(t), \quad t \in[0, T]
$$

Since $\boldsymbol{x}^{\delta}$ and $\boldsymbol{x}^{0}$ are continuous, $\boldsymbol{v}^{\delta}$ is also continuous.

Now, it follows from equations (1)-(2) that for each $\delta \in \mathbb{R}$,

$$
\boldsymbol{x}^{\delta}(t)=\boldsymbol{\varphi}\left(\hat{\boldsymbol{\sigma}}+\delta \boldsymbol{e}^{k}\right)+\int_{0}^{t} \boldsymbol{f}\left(s, \boldsymbol{x}^{\delta}(s), \boldsymbol{u}(s), \hat{\boldsymbol{\sigma}}+\delta \boldsymbol{e}^{k}\right) d s, \quad t \in[0, T] .
$$

Thus,

$$
\begin{aligned}
\boldsymbol{v}^{\delta}(t)=\boldsymbol{\varphi}\left(\hat{\boldsymbol{\sigma}}+\delta \boldsymbol{e}^{k}\right)-\boldsymbol{\varphi}(\hat{\boldsymbol{\sigma}})+\int_{0}^{t}\left\{\boldsymbol{f}\left(s, \boldsymbol{x}^{\delta}(s), \boldsymbol{u}(s), \hat{\boldsymbol{\sigma}}+\delta \boldsymbol{e}^{k}\right)\right. \\
\left.-\boldsymbol{f}\left(s, \boldsymbol{x}^{0}(s), \boldsymbol{u}(s), \hat{\boldsymbol{\sigma}}\right)\right\} d s, \quad t \in[0, T]
\end{aligned}
$$


Consequently, by the mean value theorem,

$$
\begin{aligned}
\boldsymbol{v}^{\delta}(t)=\int_{0}^{1} & \delta \frac{\partial \boldsymbol{\varphi}\left(\hat{\boldsymbol{\sigma}}+\eta \delta \boldsymbol{e}^{k}\right)}{\partial \sigma_{k}} d \eta \\
& +\int_{0}^{t} \int_{0}^{1}\left\{\frac{\partial \boldsymbol{f}\left(s, \boldsymbol{x}^{0}(s)+\eta \boldsymbol{v}^{\delta}(s), \boldsymbol{u}(s), \hat{\boldsymbol{\sigma}}+\eta \delta \boldsymbol{e}^{k}\right)}{\partial \boldsymbol{x}} \boldsymbol{v}^{\delta}(s)\right. \\
& \left.+\delta \frac{\partial \boldsymbol{f}\left(s, \boldsymbol{x}^{0}(s)+\eta \boldsymbol{v}^{\delta}(s), \boldsymbol{u}(s), \hat{\boldsymbol{\sigma}}+\eta \delta \boldsymbol{e}^{k}\right)}{\partial \sigma_{k}}\right\} d \eta d s, \quad t \in[0, T] .
\end{aligned}
$$

This identity will be used later in the proof.

Now, because of Assumption 2, it follows from Lemma 6.4.2 of [12] that the family of state trajectories

$$
\left\{\boldsymbol{x}^{\delta}: \delta \in[-1,1]\right\}
$$

is equibounded on $[0, T]$. Hence, there exists a real number $L_{2}>0$ such that for each $\delta \in[-1,1]$,

$$
\boldsymbol{x}^{\delta}(t) \in \mathcal{B}_{n}\left(L_{2}\right), \quad t \in[0, T],
$$

where $\mathcal{B}_{n}\left(L_{2}\right)$ denotes the closed ball in $\mathbb{R}^{n}$ of radius $L_{2}$ centered at the origin. Since $\mathcal{B}_{n}\left(L_{2}\right)$ is convex, for each $\delta \in[-1,1]$ we have

$$
\boldsymbol{x}^{0}(t)+\eta \boldsymbol{v}^{\delta}(t) \in \mathcal{B}_{n}\left(L_{2}\right), \quad t \in[0, T], \quad \eta \in[0,1] .
$$

Moreover, it's obvious that for each $\delta \in[-1,1]$,

$$
\hat{\boldsymbol{\sigma}}+\eta \delta \boldsymbol{e}^{k} \in \mathcal{B}_{m}\left(L_{3}\right), \quad \eta \in[0,1],
$$

where $L_{3} \triangleq|\hat{\boldsymbol{\sigma}}|_{m}+1$. Recall from Assumption 1 that $\partial \boldsymbol{\varphi} / \partial \sigma_{k}, \partial \boldsymbol{f} / \partial \sigma_{k}$, and $\partial \boldsymbol{f} / \partial \boldsymbol{x}$ are continuous. Hence, it follows from inclusions (A.3) and (A.4) and the compactness of $[0, T], \mathcal{B}_{n}\left(L_{2}\right), \mathcal{W}$, and $\mathcal{B}_{m}\left(L_{3}\right)$ that there exists a real number $L_{4}>0$ such that for each $\delta \in[-1,1]$,

$$
\begin{gathered}
\left|\frac{\partial \boldsymbol{\varphi}\left(\hat{\boldsymbol{\sigma}}+\eta \delta \boldsymbol{e}^{k}\right)}{\partial \sigma_{k}}\right|_{n} \leq L_{4}, \quad \eta \in[0,1], \\
\left|\frac{\partial \boldsymbol{f}\left(t, \boldsymbol{x}^{0}(t)+\eta \boldsymbol{v}^{\delta}(t), \boldsymbol{u}(t), \hat{\boldsymbol{\sigma}}+\eta \delta \boldsymbol{e}^{k}\right)}{\partial \sigma_{k}}\right|_{n} \leq L_{4}, \quad t \in[0, T], \quad \eta \in[0,1], \\
\left|\frac{\partial \boldsymbol{f}\left(t, \boldsymbol{x}^{0}(t)+\eta \boldsymbol{v}^{\delta}(t), \boldsymbol{u}(t), \hat{\boldsymbol{\sigma}}+\eta \delta \boldsymbol{e}^{k}\right)}{\partial \boldsymbol{x}}\right|_{n \times n} \leq L_{4}, \quad t \in[0, T], \quad \eta \in[0,1] .
\end{gathered}
$$

Step 2. The function $\boldsymbol{v}^{\delta}$ is of order $\delta$

Let $\delta \in[-1,1]$ be arbitrary. Taking the norm of both sides of (A.2) and applying the definition of $L_{4}$ gives

$$
\left|\boldsymbol{v}^{\delta}(t)\right|_{n} \leq L_{4}|\delta|+L_{4} T|\delta|+\int_{0}^{t} L_{4}\left|\boldsymbol{v}^{\delta}(s)\right|_{n} d s, \quad t \in[0, T] .
$$


Applying Gronwall's Lemma then gives

$$
\left|\boldsymbol{v}^{\delta}(t)\right|_{n} \leq\left(L_{4}+L_{4} T\right) \exp \left(L_{4} T\right)|\delta|, \quad t \in[0, T],
$$

and since $\delta \in[-1,1]$ was arbitrary, this inequality holds whenever the magnitude of $\delta$ is sufficiently small (less than or equal to one). Thus, the function $\boldsymbol{v}^{\delta}$ is of order $\delta$.

Step 3. Definition and limiting behavior of $\rho_{1}$

Define a function $\rho_{1}:[-1,1] \rightarrow \mathbb{R}$ as follows:

$$
\rho_{1}(\delta) \triangleq \int_{0}^{1}\left|\frac{\partial \boldsymbol{\varphi}\left(\hat{\boldsymbol{\sigma}}+\eta \delta \boldsymbol{e}^{k}\right)}{\partial \sigma_{k}}-\frac{\partial \boldsymbol{\varphi}(\hat{\boldsymbol{\sigma}})}{\partial \sigma_{k}}\right|_{n} d \eta, \quad \delta \in[-1,1] .
$$

Clearly $\hat{\boldsymbol{\sigma}}+\eta \delta \boldsymbol{e}^{k} \rightarrow \hat{\boldsymbol{\sigma}}$ as $\delta \rightarrow 0$, uniformly with respect to $\eta \in[0,1]$. This convergence takes place inside the ball $\mathcal{B}_{m}\left(L_{3}\right)$ (see inclusion (A.4) in Step 1). Hence, since $\partial \varphi / \partial \sigma_{k}$ is uniformly continuous on the compact set $\mathcal{B}_{m}\left(L_{3}\right)$,

$$
\frac{\partial \boldsymbol{\varphi}\left(\hat{\boldsymbol{\sigma}}+\eta \delta \boldsymbol{e}^{k}\right)}{\partial \sigma_{k}} \rightarrow \frac{\partial \boldsymbol{\varphi}(\hat{\boldsymbol{\sigma}})}{\partial \sigma_{k}} \quad \text { as } \quad \delta \rightarrow 0,
$$

uniformly with respect to $\eta \in[0,1]$. Consequently,

$$
\lim _{\delta \rightarrow 0} \rho_{1}(\delta)=0 .
$$

Step 4. Definition and limiting behavior of $\rho_{2}$

For each $\delta \in \mathbb{R}$, define two corresponding functions $\boldsymbol{\lambda}^{1, \delta}:[0, T] \rightarrow \mathbb{R}^{n}$ and $\boldsymbol{\lambda}^{2, \delta}:[0, T] \rightarrow \mathbb{R}^{n}$ as follows:

$$
\begin{aligned}
& \lambda^{1, \delta}(t) \triangleq \int_{0}^{1}\left\{\frac{\partial \boldsymbol{f}\left(t, \boldsymbol{x}^{0}(t)+\eta \boldsymbol{v}^{\delta}(t), \boldsymbol{u}(t), \hat{\boldsymbol{\sigma}}+\eta \delta \boldsymbol{e}^{k}\right)}{\partial \boldsymbol{x}}\right. \\
&\left.-\frac{\partial \boldsymbol{f}\left(t, \boldsymbol{x}^{0}(t), \boldsymbol{u}(t), \hat{\boldsymbol{\sigma}}\right)}{\partial \boldsymbol{x}}\right\} \boldsymbol{v}^{\delta}(t) d \eta, \quad t \in[0, T],
\end{aligned}
$$

and

$$
\begin{aligned}
& \boldsymbol{\lambda}^{2, \delta}(t) \triangleq \int_{0}^{1} \delta\left\{\frac{\partial \boldsymbol{f}\left(t, \boldsymbol{x}^{0}(t)+\eta \boldsymbol{v}^{\delta}(t), \boldsymbol{u}(t), \hat{\boldsymbol{\sigma}}+\eta \delta \boldsymbol{e}^{k}\right)}{\partial \sigma_{k}}\right. \\
&\left.-\frac{\partial \boldsymbol{f}\left(t, \boldsymbol{x}^{0}(t), \boldsymbol{u}(t), \hat{\boldsymbol{\sigma}}\right)}{\partial \sigma_{k}}\right\} d \eta, \quad t \in[0, T] .
\end{aligned}
$$

Furthermore, define another function $\rho_{2}:[-1,0) \cup(0,1] \rightarrow \mathbb{R}$ as follows:

$$
\rho_{2}(\delta) \triangleq|\delta|^{-1} \int_{0}^{T}\left\{\left|\boldsymbol{\lambda}^{1, \delta}(t)\right|_{n}+\left|\boldsymbol{\lambda}^{2, \delta}(t)\right|_{n}\right\} d t, \quad \delta \in[-1,0) \cup(0,1] .
$$

Now, it follows immediately from (A.5) that

$$
\boldsymbol{x}^{0}(t)+\eta \boldsymbol{v}^{\delta}(t) \rightarrow \boldsymbol{x}^{0}(t) \quad \text { as } \quad \delta \rightarrow 0,
$$


uniformly with respect to $t \in[0, T]$ and $\eta \in[0,1]$. In addition, recall from Step 3 that

$$
\hat{\boldsymbol{\sigma}}+\eta \delta \boldsymbol{e}^{k} \rightarrow \hat{\boldsymbol{\sigma}} \quad \text { as } \quad \delta \rightarrow 0,
$$

uniformly with respect to $\eta \in[0,1]$. Since the convergence in (A.7) takes place inside the ball $\mathcal{B}_{n}\left(L_{2}\right)$, the convergence in (A.8) takes place inside the ball $\mathcal{B}_{m}\left(L_{3}\right)$, and $\partial \boldsymbol{f} / \partial \boldsymbol{x}$ and $\partial \boldsymbol{f} / \partial \sigma_{k}$ are uniformly continuous on the compact set $[0, T] \times \mathcal{B}_{n}\left(L_{2}\right) \times \mathcal{W} \times \mathcal{B}_{m}\left(L_{3}\right)$,

$$
\frac{\partial \boldsymbol{f}\left(t, \boldsymbol{x}^{0}(t)+\eta \boldsymbol{v}^{\delta}(t), \boldsymbol{u}(t), \hat{\boldsymbol{\sigma}}+\eta \delta \boldsymbol{e}^{k}\right)}{\partial \boldsymbol{x}} \rightarrow \frac{\partial \boldsymbol{f}\left(t, \boldsymbol{x}^{0}(t), \boldsymbol{u}(t), \hat{\boldsymbol{\sigma}}\right)}{\partial \boldsymbol{x}} \quad \text { as } \quad \delta \rightarrow 0
$$

and

$$
\frac{\partial \boldsymbol{f}\left(t, \boldsymbol{x}^{0}(t)+\eta \boldsymbol{v}^{\delta}(t), \boldsymbol{u}(t), \hat{\boldsymbol{\sigma}}+\eta \delta \boldsymbol{e}^{k}\right)}{\partial \sigma_{k}} \rightarrow \frac{\partial \boldsymbol{f}\left(t, \boldsymbol{x}^{0}(t), \boldsymbol{u}(t), \hat{\boldsymbol{\sigma}}\right)}{\partial \sigma_{k}} \quad \text { as } \quad \delta \rightarrow 0,
$$

uniformly with respect to $t \in[0, T]$ and $\eta \in[0,1]$. These results, together with inequality (A.5), imply that $\delta^{-1} \boldsymbol{\lambda}^{1, \delta} \rightarrow \mathbf{0}$ and $\delta^{-1} \boldsymbol{\lambda}^{2, \delta} \rightarrow \mathbf{0}$ uniformly on $[0, T]$ as $\delta \rightarrow 0$. Consequently,

$$
\lim _{\delta \rightarrow 0} \rho_{2}(\delta)=0 .
$$

Step 5. The final step: Comparing $\delta^{-1} \boldsymbol{v}^{\delta}$ with $\boldsymbol{\psi}^{k}(\cdot \mid \boldsymbol{u})$

We now use the results proved in the previous steps to establish equation (A.1). First, let $\delta \in[-1,0) \cup(0,1]$ be arbitrary but fixed. Next, using (A.2) we obtain

$$
\begin{aligned}
\boldsymbol{v}^{\delta}(t)= & \delta \frac{\partial \boldsymbol{\varphi}(\hat{\boldsymbol{\sigma}})}{\partial \sigma_{k}}+\int_{0}^{1} \delta\left\{\frac{\partial \boldsymbol{\varphi}\left(\hat{\boldsymbol{\sigma}}+\eta \delta \boldsymbol{e}^{k}\right)}{\partial \sigma_{k}}-\frac{\partial \boldsymbol{\varphi}(\hat{\boldsymbol{\sigma}})}{\partial \sigma_{k}}\right\} d \eta \\
& +\int_{0}^{t}\left(\boldsymbol{\lambda}^{1, \delta}(s)+\boldsymbol{\lambda}^{2, \delta}(s)\right) d s+\int_{0}^{t} \frac{\partial \boldsymbol{f}\left(s, \boldsymbol{x}^{0}(s), \boldsymbol{u}(s), \hat{\boldsymbol{\sigma}}\right)}{\partial \boldsymbol{x}} \boldsymbol{v}^{\delta}(s) d s \\
& +\int_{0}^{t} \delta \frac{\partial \boldsymbol{f}\left(s, \boldsymbol{x}^{0}(s), \boldsymbol{u}(s), \hat{\boldsymbol{\sigma}}\right)}{\partial \sigma_{k}} d s, \quad t \in[0, T]
\end{aligned}
$$

Furthermore, integrating the auxiliary system gives

$$
\begin{aligned}
\boldsymbol{\psi}^{k}(t \mid \boldsymbol{u})=\frac{\partial \boldsymbol{\varphi}(\hat{\boldsymbol{\sigma}})}{\partial \sigma_{k}}+ & \int_{0}^{t} \frac{\partial \boldsymbol{f}\left(s, \boldsymbol{x}^{0}(s), \boldsymbol{u}(s), \hat{\boldsymbol{\sigma}}\right)}{\partial \boldsymbol{x}} \boldsymbol{\psi}^{k}(s \mid \boldsymbol{u}) d s \\
& +\int_{0}^{t} \frac{\partial \boldsymbol{f}\left(s, \boldsymbol{x}^{0}(s), \boldsymbol{u}(s), \hat{\boldsymbol{\sigma}}\right)}{\partial \sigma_{k}} d s, \quad t \in[0, T] .
\end{aligned}
$$

Multiplying equation (A.10) by $\delta^{-1}$ and then subtracting from it equation (A.11) gives

$$
\begin{aligned}
& \delta^{-1} \boldsymbol{v}^{\delta}(t)-\boldsymbol{\psi}^{k}(t \mid \boldsymbol{u})=\int_{0}^{1}\left\{\frac{\partial \boldsymbol{\varphi}\left(\hat{\boldsymbol{\sigma}}+\eta \delta \boldsymbol{e}^{k}\right)}{\partial \sigma_{k}}\right.\left.-\frac{\partial \boldsymbol{\varphi}(\hat{\boldsymbol{\sigma}})}{\partial \sigma_{k}}\right\} d \eta \\
&+\delta^{-1} \int_{0}^{t}\left(\boldsymbol{\lambda}^{1, \delta}(s)+\boldsymbol{\lambda}^{2, \delta}(s)\right) d s \\
&+\int_{0}^{t} \frac{\partial \boldsymbol{f}\left(s, \boldsymbol{x}^{0}(s), \boldsymbol{u}(s), \hat{\boldsymbol{\sigma}}\right)}{\partial \boldsymbol{x}}\left(\delta^{-1} \boldsymbol{v}^{\delta}(s)-\boldsymbol{\psi}^{k}(s \mid \boldsymbol{u})\right) d s, \quad t \in[0, T] .
\end{aligned}
$$


Therefore,

$\left|\delta^{-1} \boldsymbol{v}^{\delta}(t)-\boldsymbol{\psi}^{k}(t \mid \boldsymbol{u})\right|_{n} \leq \rho_{1}(\delta)+\rho_{2}(\delta)+\int_{0}^{t} L_{4}\left|\delta^{-1} \boldsymbol{v}^{\delta}(s)-\boldsymbol{\psi}^{k}(s \mid \boldsymbol{u})\right|_{n} d s, t \in[0, T]$.

By Gronwall's Lemma,

$$
\left|\delta^{-1} \boldsymbol{v}^{\delta}(t)-\boldsymbol{\psi}^{k}(t \mid \boldsymbol{u})\right|_{n} \leq\left(\rho_{1}(\delta)+\rho_{2}(\delta)\right) \exp \left(L_{4} T\right), \quad t \in[0, T] .
$$

Recalling that $\delta \in[-1,0) \cup(0,1]$ was arbitrary, we can take the limit as $\delta \rightarrow 0$ in the above inequality and apply (A.6) and (A.9) to establish

$$
\lim _{\delta \rightarrow 0} \delta^{-1} \boldsymbol{v}^{\delta}(t)=\boldsymbol{\psi}^{k}(t \mid \boldsymbol{u}), \quad t \in[0, T] .
$$

This proves equation (A.1), because $\boldsymbol{v}^{\delta}(t)=\boldsymbol{x}\left(t \mid \boldsymbol{u}, \hat{\boldsymbol{\sigma}}+\delta \boldsymbol{e}^{k}\right)-\boldsymbol{x}(t \mid \boldsymbol{u}, \hat{\boldsymbol{\sigma}})$ for each $t \in[0, T]$.

\section{References}

[1] N. U. Ahmed, Dynamic Systems and Control with Applications, World Scientific, Singapore, 2006.

[2] C. Büskens, H. Maurer, SQP-methods for solving optimal control problems with control and state constraints: adjoint variables, sensitivity analysis and real-time control, Journal of Computational and Applied Mathematics 120 (2000) 85-108.

[3] M. E. Fisher, W. J. Grantham, K. L. Teo, Neighbouring extremals for nonlinear systems with control constraints, Dynamics and Control 5 (1995) 225-240.

[4] S. Gonzalez, A. Miele, Sequential gradient-restoration algorithm for optimal control problems with general boundary conditions, Journal of Optimization Theory and Applications 26 (1978) 395-425.

[5] W. W. Hager, Runge-Kutta methods in optimal control and the transformed adjoint system, Numerische Mathematik 87 (2000) 247-282.

[6] L. S. Jennings, M. E. Fisher, K. L. Teo, C. J. Goh, MISER3 Optimal Control Software: Theory and User Manual, University of Western Australia, Perth, 2004.

[7] C. Y. Kaya, J. M. Martínez, Euler discretization and inexact restoration for optimal control, Journal of Optimization Theory and Applications 134 (2007) 191-206.

[8] R. Loxton, K. L. Teo, V. Rehbock, Optimal control problems with multiple characteristic time points in the objective and constraints, Automatica 44 (2008) 2923-2929. 
[9] A. Miele, R. E. Pritchard, J. N. Damoulakis, Sequential gradientrestoration algorithm for optimal control problems, Journal of Optimization Theory and Applications 5 (1970) 235-282.

[10] N. B. Nedeljković, New algorithms for unconstrained nonlinear optimal control problems, IEEE Transactions on Automatic Control 26 (1981) 868884 .

[11] V. Rehbock, K. L. Teo, L. S. Jennings, A computational procedure for suboptimal robust controls, Dynamics and Control 2 (1992) 331-348.

[12] K. L. Teo, C. J. Goh, K. H. Wong, A Unified Computational Approach to Optimal Control Problems, Longman Scientific and Technical, Essex, 1991.

[13] K. L. Teo, L. S. Jennings, H. W. J. Lee, V. Rehbock, The control parameterization enhancing transform for constrained optimal control problems, Journal of the Australian Mathematical Society-Series B 40 (1999) 314335 .

[14] O. von Stryk, Numerical solution of optimal control problems by direct collocation, in: R. Bulirsch (Eds.), Optimal Control-Calculus of Variations, Optimal Control Theory and Numerical Methods, Birkhäuser, Basel, 1993, pp. 129-143. 